\section{Summary of: Dental age assessment (DAA): a simple method for children and emerging adults}

\author{
G. J. Roberts, ${ }^{1}$ S. Parekh, ${ }^{2}$ A. Petrie ${ }^{3}$ and V. S. Lucas ${ }^{4}$
}

\section{FULL PAPER DETAILS}

1"Professor and Consultant in Paediatric Dentistry, King's College London Dental Institute, Bessemer Road, London, SE5 9RS; ${ }^{2}$ Lecturer in Paediatric Dentistry, UCL Eastman Dental Institute, 256 Gray's Inn Road, London, WCX 8LD; ${ }^{3}$ Senior Lecturer and Head, Unit of Biostatistics, UCL Eastman Dental Institute, 256 Gray's Inn Road, London, WC1X 8LD; ${ }^{4}$ Senior Clinical Research Fellow, King's College London Dental Institute, Bessemer Road, London, SE5 9RS ${ }^{*}$ Correspondence to: Professor Graham J. Roberts Email: graham.j.roberts@kcl.ac.uk

Online article number E7

Refereed Paper - accepted 24 April 2007

DOI: $10.1038 /$ bdj.2008.21

${ }^{\circ}$ British Dental Journal 2008; 204: E7

Introduction Methods of dental age assessment (DAA) give a wide margin of error and, because third molars are usually excluded, prevent estimation around the age of 18 years. This study extends the use of defined tooth development stages (TDSs) to include third molars. Subjects and methods Re-use of dental panoramic tomographs (DPTs) and other $X$-rays taken for clinical use comprised the sample of 1,547 subjects. The radiographic images were then captured in digital format. The TDSs were assessed and the estimated mean age and its standard error were calculated for each TDS. The mathematical technique of meta-analysis was used to provide an estimate of the mean age, with 99\% confidence interval, of a new 'test' subject. To assess the accuracy of the method, each of these mean values was then compared with the gold standard of chronological age. Results On average, estimated dental age (DA) over-estimated chronological age (CA) by 0.29 years, approximately $31 \frac{1}{2}$ months. The maximum likely difference between the estimated DA and CA was 1.65 years. Conclusion Estimation of dental age using well defined TDSs, extended to include third molars and combined with the statistical technique of meta-analysis, provides investigators with a rapid and accurate estimation of age.

\section{EDITOR'S SUMMARY}

The easiest way to discover someone's age is, of course, to ask them. However, you may not get an answer or the answer that you get may not be correct or accurate. To begin with the person may not understand your question, they may be too young and/or they may not speak the same language or they may have a mental incapacity such as a learning disability. Assuming they do not fall into any of these categories and can answer, they may not know their precise age as they have no documentary evidence to support it, or they may know it but seek to obscure it for a variety of reasons. There is one other rather less pleasant reason - they may be deceased.

In any of these situations the use of the teeth as a measure of age has been adopted since the dawn of history. This has not always been to the advantage of the individual, sometimes having been used to define the age at which conscription, or suitability for work may take place. Until now the accuracy of this process has been less than precise, or perhaps more pertinently, less precise than we would like. However, this paper has pulled together data from other studies and by meta-analysis, pooled the results. The outcome is a system which, pending use in practice, could provide the most certain measure yet of chronological age of an individual.

Before the availability of radiographs, estimation could only be made on the basis of observation of erupted teeth. The availability of radiography does however mean that one note of caution needs to be sounded and the authors have alluded to this in their methodology. That is, that the radiographs used in this study were 're-used' after they had been taken for clinical use. The ethics of taking radiographs purely for the identification of age has to be closely questioned since any exposure to radiation brings with it a health risk to the individual. In the clinical situation, this risk must be balanced by a benefit to the patient. So too must a need to pro- vide proof of the age of an individual by use of radiography be argued on an advantage to that person and not on the basis of a convenience for an investigating authority, however compelling it may seem.

The full paper can be accessed from the $B D J$ website (www.bdj.co.uk), under 'Research' in the table of contents for Volume 204 issue 4.

Stephen Hancocks, Editor-in-Chief

DOI: $10.1038 /$ bdj.2008.148 


\section{TO ACCESS THE BDJ WEBSITE TO READ THE FULL PAPER:}

- BDA members should go to www.bda.org

- Do not login on the BDA home page, if you are already logged in, please log out.

- Then, in www.bda.org click on the link to the BDJ in the top left of the screen. A new window will open. If a new window fails to open please check the settings of any pop up blocker software that you have installed on your computer.

- You will now be asked to login with your BDA website login details which are on your BDA membership card.

- Once your details have been entered you will be transferred to the BDJ website. If your login does not work please contact the BDA Membership Department on 02075634550.

- If you are not able to access the article on the BDJ website there may be an issue with your system's firewall. If so, return to the BDA homepage and click on the link 'BDJ access problems' and follow the step by step guide.

\section{COMMENT}

With the change in both social and demographic strata of our society, and increased mobility of individuals and the requirements of legal precision,* governments are called upon to determine the biological age of patients, victims of crimes such as abduction coupled with abuse, and asylum seekers.

The most reliable method of assessing age is the state of the development of teeth and in common with other investigators, it is the specific age of attainment and the numerical summary data for the tooth development stages (TDSs) that are important in estimating age. The integration of the differing ages for each of the TDSs present has always been dealt with in a somewhat unsatisfactory way. This study reports the use of the mathematical techniques of meta-analysis to calculate the age of an individual subject with 99\% confidence intervals. This is a significant advance as reliable numerical assessments can be made. As is shown in the paper, the average age estimation is usually accurate to within 3.5 months and it is important to note that this comparison is with the gold standard of known and verifiable chronological age. This is suitable for civil law proceedings where the acceptable underlying assumption is that the subject under scrutiny is of average growth and development. The authors have also provided an estimate of the extremes of variation and have shown that the difference between estimated age and chronological age could be as great as 1.65 years, an appropriate cautionary note.

This paper provides a novel, unique and simple method which is very practical when performed by any clinician with only a limited understanding of computer-based algorithms. As with any new approach to a clinical problem, other investigators need to explore the ramifications of this technique to further clarify its value in DAA. For the moment, these investigators have made a significant advance with this simple and reliable way of estimating the age of subjects of unknown (or unrevealed) date of birth. Simple solutions are always so easy to recognise after someone else has identified them.

\section{F. McDonald, Kings College London Dental Institute}

*The law has very specific requirements about the sentencing of youths in particular; the age of an offender is specifically defined in order to identify the sentencing and recommendations.

\section{AUTHOR QUESTIONS AND ANSWERS}

1. Why did you undertake this research? I have always had an interest in growth and development and as part this, the relationship between growth standards and age. In 2004, the number of requests for dental age assessment from solicitors acting on behalf of subjects without birth records as such, minors or asylum seekers appeared to increase. The concern was that solicitors would enquire as to the accuracy of the methods we used. (At that time we were using dental anatomy texts). It was necessary to develop a method of utilising age specific data to provide lawyers with a reliable way of estimating age with appropriate confidence intervals.

2. What would you like to do next in this area to follow on from this work?

To develop the statistical methods of dental age assessment utilising the final stage of root development of third molars (apical closure), a unique challenge for statistical methodology. 